\title{
Qualidade da madeira acetilada de Eucalyptus urophylla x Eucalyptus grandis
}

\author{
Gustavo Gomes Figueiredo ${ }^{1}$ Diego Martins Stangerlin ${ }^{1 *}$ Mayra Daniela Ferreira ${ }^{2}$ Leonardo Antônio \\ Moraes Zaque ${ }^{2}$ Elisangela Pariz ${ }^{1}$ Bárbara Luísa Corradi Pereira ${ }^{2}$
}

${ }^{1}$ Universidade Federal do Mato Grosso, Av. Alexandre Ferronato, 1200, St. Industrial, CEP 78557-267, Sinop, MT, Brasil

${ }^{2}$ Universidade Federal do Mato Grosso, Av. Fernando Corrêa da Costa, 2367, Boa Esperança, CEP 78060-900, Cuiabá, MT, Brasil

\begin{abstract}
Original Article
*Corresponding author: stangerlin@ufmt.br

Palavras-chave:

Modificação química

Acetilação

Qualidade da madeira

Keywords:

Chemical modification

Acetylation

RESUMO: Objetivou-se com este estudo avaliar as propriedades tecnológicas da madeira de Eucalyptus urophylla $\mathrm{x}$ Eucalpyptus grandis submetida ao processo de modificação química por acetilação. Para tanto, foram confeccionadas amostras de madeira com dimensões de $2 \times 2 \times 1 \mathrm{~cm}$, as quais foram submetidas ao tratamento de acetilação, por imersão em anidrido acético, durante 3 e 6 h com temperatura de $75^{\circ} \mathrm{C}$. A eficiência da modificação química foi avaliada por meio da determinação de propriedades da madeira acetilada e controle (não tratada): massa específica aparente, absorção em água, inchamento volumétrico, dureza Rockwell e resistência ao ataque de cupins. A acetilação da madeira proporcionou aumento da estabilidade dimensional, resistência biológica e mecânica, quando comparadas às amostras não tratadas. Por outro lado, não foi verificada alteração na massa específica aparente, em condições anidras, das madeiras acetiladas e controle.
\end{abstract}

Wood quality

Received in

2019/06/27

Accepted on

2020/02/14

Published in

2020/03/04

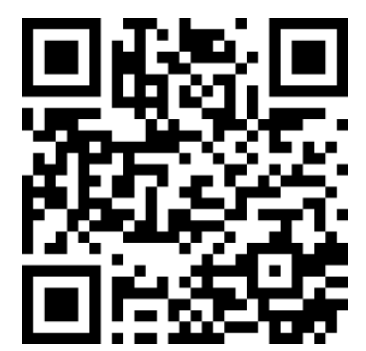

DOI:http://dx.doi.org/10.340 62/afs.v7i1.8559

\section{(cc) BY}

\section{Quality of acetylated wood of Eucalyptus urophylla $\mathbf{x}$ Eucalyptus grandis}

\begin{abstract}
The present study aimed to evaluate the technological properties of Eucalyptus urophylla $\mathrm{x}$ Eucalyptus grandis wood submitted to the chemical modification process by acetylation. For this, $2 \times 2 \times 1 \mathrm{~cm}$ wood samples were prepared, which were submitted to the acetylation treatment by immersion in acetic anhydride for 3 and $6 \mathrm{~h}$ at a temperature of $75^{\circ} \mathrm{C}$. The chemical modification efficiency was evaluated by determining the properties of acetylated and control (untreated) wood: apparent specific gravity, water absorption, volumetric swelling, Rockwell hardness and resistance to termite attack. Wood acetylation provided increased dimensional stability, biological and mechanical resistance when compared to untreated samples. On the other hand, there was no change in the apparent specific gravity, under anhydrous conditions, of the acetylated and control woods.
\end{abstract}




\section{Introdução}

A modificação da madeira visa melhorar algumas de suas propriedades tecnológicas, tais como a resistência à biodegradação, a estabilidade dimensional, entre outras (Esteves, Pereira, 2009). Grande parte dos processos de modificação química baseia-se na reação dos grupos hidroxila da madeira e um reagente químico. Ao substituir estes grupos por um composto hidrofóbico, ocorre uma redução na higroscopicidade da madeira, resultando em um material com propriedades melhoradas (Esteves et al., 2011). Um dos métodos mais promissores de modificação química é a esterificação da madeira. Nesta, a mais estudada é a acetilação com anidrido acético, que já é utilizada comercialmente em diversos países ao redor do mundo (Homan e Bongers, 2004; Rowell, 2014).

$\mathrm{Na}$ reação com anidrido acético, os grupos hidroxílicos presentes nos constituintes da parede celular da madeira são convertidos em grupos acetato, que por sua vez apresentam característica hidrofóbica. Como subproduto desta reação, há a formação do ácido acético (Homan et al., 2000; Rowell, 2014). Isto implica na obstrução dos grupos hidrófilos da madeira, o que conduz a uma menor afinidade deste material com a água, proporcionando um aumento na sua estabilidade dimensional (Castro, Iwakiri, 2014).

No Brasil, emprega-se ainda um baixo volume de madeira tratada, quando comparado à países do continente Europeu e Norte Americano. Entretanto, o país apresenta grande potencial de expansão rumo a novos mercados emergentes, como o da construção civil, embalagens e linhas de transmissão (cruzetas e postes). Os principais métodos de tratamento preservativo da madeira no Brasil são aqueles realizados sob pressão, em autoclave, utilizando o método Bethell. Estes tratamentos utilizam preservativos oleosos e hidrossolúveis, e são amplamente aplicados em espécies do gênero Eucalyptus e Pinus (Vidal et al., 2015).

As espécies do gênero Eucalyptus se destacam por apresentar rápido crescimento, sobretudo nas condições tropicais, o que confere ao Brasil uma vantagem competitiva em relação a outros países concorrentes, devido às condições favoráveis de clima, solo, extensão territorial, mão de obra, infraestrutura e capacidade gerencial produtiva (Valverde et al., 2004).

Atualmente no exterior, sobretudo na Europa, o tratamento de acetilação já vem sendo utilizado em escala industrial, e os produtos oriundos deste processo tem sido inseridos no mercado com grande valor agregado (Rowell, 2013). Entretanto, no Brasil este método ainda se encontra em estágio de pesquisa, com resultados ainda escassos.

Face ao exposto, objetivou-se com este trabalho avaliar o efeito da acetilação nas propriedades tecnológicas da madeira de Eucalyptus urophylla x Eucalyptus grandis.

\section{Material e Métodos \\ Coleta e preparo das amostras}

Neste trabalho foram utilizadas madeiras de Eucalyptus urophylla $\mathrm{x}$ Eucalyptus grandis oriundas de um plantio homogêneo, implantado no município de Sinop-MT em espaçamento de 3 x 3,5 m, com 5 anos de idade. Para tanto, foram abatidas quatro árvores, a partir das quais foi selecionada a primeira tora (até $1,3 \mathrm{~m}$ ) para confecção de 90 amostras com dimensões nominais de 2,0 × 2,0 x $1,0 \mathrm{~cm}$ (sendo a menor dimensão no sentido axial).

As amostras foram acondicionadas em estufa de circulação forçada de ar a uma temperatura de $60^{\circ}$ $\mathrm{C}$, até o momento em que atingiram a condição de massa e dimensões anidras. Ao final da secagem, as amostras de madeira foram divididas em três grupos com 30 amostras cada: controle (não acetilada), acetiladas por 3 e 6 horas.

\section{Tratamento de acetilação}

Para o tratamento de acetilação foi utilizada a metodologia adaptada por Gomes (2005). As amostras foram submetidas ao tratamento por dois períodos de tempo -3 e $6 \mathrm{~h}$ - com temperatura constante de $75^{\circ} \mathrm{C}$. Para tanto, as amostras foram imersas em dois béqueres de $1000 \mathrm{~mL}$ contendo anidrido acético, e então o material foi mantido em aquecimento de banho-maria.

Após os períodos de 3 e $6 \mathrm{~h}$, as amostras foram retiradas do banho-maria e lavadas com etanol, a fim de retirar o excesso de reagente. As amostras foram então submetidas à secagem em estufa de circulação forçada de ar a $60^{\circ} \mathrm{C}$, até atingirem massa e dimensões anidras.

\section{Ganho de massa e massa específica aparente}

$\mathrm{O}$ ganho de massa das amostras resultante do processo de acetilação foi determinado por meio da relação entre as massas secas antes e após o tratamento ser realizado (Equação 1). Já a massa específica aparente foi determinada através da relação entre a massa e o volume em condições anidras.

$$
\mathrm{GM}=\left(\frac{\mathrm{Mt}-\mathrm{Mnt}}{\mathrm{Mnt}}\right) * 100 \quad(\text { Equação } 1)
$$

em que: GM: ganho de massa (\%); Mt: massa seca da madeira tratada (g); Mnt: massa seca da madeira não tratada $(\mathrm{g})$.

\section{Absorção em água e inchamento volumétrico}

A determinação da absorção em água (Equação 2) e inchamento volumétrico (Equação 3) foi realizada de acordo com os procedimentos estabelecidos pela NBR 7190 da Associação Brasileira de Normas Técnicas - ABNT (1997). 
Para tanto, utilizaram-se 10 amostras de cada tratamento (controle, 3 e $6 \mathrm{~h}$ de acetilação), as quais foram imersas em água destilada, até a saturação total, aproximadamente 30 dias. Após esse período, determinaram-se a massa e o volume das amostras saturadas.

$$
\mathrm{ABS}=\left(\frac{\mathrm{Mu}-\mathrm{Ms}}{\mathrm{Ms}}\right) * 100 \quad(\text { Equação } 2)
$$

em que: ABS: absorção em água (\%); Um: massa úmida (g); Ms: massa seca (g).

$$
\mathrm{IV}=\left(\frac{\mathrm{Vu}-\mathrm{Vs}}{\mathrm{Vs}}\right) * 100 \quad(\text { Equação 3) }
$$

em que: IV: inchamento volumétrico (\%); Vu: volume úmido $\left(\mathrm{mm}^{3}\right)$; Vs: volume seco $\left(\mathrm{mm}^{3}\right)$.

\section{Dureza Rockwell}

Para a avaliação da dureza Rockwell foi utilizada a metodologia adaptada por Stangerlin et al. (2013), a partir da utilização de um durômetro de bancada dotado de um penetrador esférico de $1 / 4$ de polegada, onde os valores de resistência à penetração foram obtidos diretamente na escala $L$.

Foram utilizadas 10 amostras de cada tratamento (controle, 3 e $6 \mathrm{~h}$ de acetilação), nas quais Tabela 1. Classificação do nível de deterioração do material submetido ao ataque de cupins. foram aplicadas uma pré-carga de $10 \mathrm{Kgf} \mathrm{e}$, posteriormente, uma carga final de $60 \mathrm{Kgf}$. Este procedimento foi realizado em duplicata, e a média entre os valores obtidos configura o valor final da dureza Rockwell das amostras.

\section{Resistência ao ataque de cupins}

Para a avaliação da resistência biológica, foram utilizadas 10 amostras de cada tratamento (controle, 3 e $6 \mathrm{~h}$ de acetilação), as quais foram expostas ao ataque de cupins xilófagos em condições laboratoriais. Para tanto, uma colônia ativa de cupins do gênero Nasutitermes sp. foi disposta em uma caixa d'água de $500 \mathrm{~L}$, contendo $10 \mathrm{~cm}$ de camada de areia em seu interior, onde as amostras foram dispostas aleatoriamente.

O ensaio foi realizado durante um período de 45 dias, ao final as amostras foram retiradas, limpas e avaliadas conforme o procedimento $3345-8$ da American Society for Testing and Materials (ASTM, 1994). Dessa forma, foram atribuídas notas a cada uma das amostras, ponderando-se os danos evidenciados em sua superfície (Tabela 1).

\begin{tabular}{cl}
\hline Nota & Avaliação do dano causado pelo agente xilófago \\
\hline 10 & Livre de qualquer patologia \\
9 & Ataque superficial, presença de poucos orifícios e sem comprometimento estrutural. \\
7 & Ataque moderado, presença de orifícios, sem comprometimento da peça. \\
4 & Ataque intenso, presença de orifícios, galerias e comprometimento parcial da peça. \\
0 & Comprometimento da peça. \\
\hline
\end{tabular}

\section{Análise estatística}

Os resultados foram avaliados estatisticamente mediante emprego da análise de variância e comparação das médias, quando significativas, pelo teste de Tukey ao nível de 5\% de probabilidade de erro.

\section{Resultados}

Ganho de massa e massa específica aparente

O tratamento de 6 horas denotou maior percentual de ganho de massa $(11,96 \%)$, diferindo significativamente do tratamento de 3 horas, indicando que o tempo influencia neste parâmetro (Tabela 2).

Tabela 2. Comparação entre os valores médios de ganho de massa e massa específica aparente das madeiras de E. urophylla x E. grandis acetiladas e controle.

\begin{tabular}{ccc}
\hline Tratamento & $\begin{array}{c}\text { Ganho de } \\
\text { massa }\end{array}$ & $\begin{array}{c}\text { Massa } \\
\text { específica }\end{array}$ \\
\hline Controle & - & $0,51 \mathrm{~g} / \mathrm{cm}^{3} \mathrm{a}$ \\
$3 \mathrm{~h}$ & $9,59 \% \mathrm{a}$ & $0,53 \mathrm{~g} / \mathrm{cm}^{3} \mathrm{a}$ \\
$6 \mathrm{~h}$ & $11,96 \% \mathrm{~b}$ & $0,54 \mathrm{~g} / \mathrm{cm}^{3} \mathrm{a}$ \\
\hline
\end{tabular}

Médias seguidas por diferentes letras minúsculas diferem estatisticamente entre si.

\section{Absorção em água e inchamento volumétrico}

Os valores observados para a absorção em água e inchamento volumétrico denotaram diferença estatística entre a madeira acetilada e controle; todavia, não houve diferença significativa entre os tratamentos de 3 e 6 horas de acetilação (Tabela 3 ).

Tabela 3. Comparação entre os valores médios de absorção em água e inchamento volumétrico até o ponto de saturação total das madeiras de $E$. urophylla $\mathrm{x}$ E. grandis acetiladas e controle.

\begin{tabular}{ccc}
\hline Tratamento & $\begin{array}{c}\text { Inchamento } \\
\text { volumétrico }\end{array}$ & $\begin{array}{c}\text { Absorção em } \\
\text { água }\end{array}$ \\
\hline Controle & $17,47 \% \mathrm{a}$ & $155,92 \% \mathrm{a}$ \\
$3 \mathrm{~h}$ & $13,41 \% \mathrm{~b}$ & $136,47 \% \mathrm{~b}$ \\
$6 \mathrm{~h}$ & $11,73 \% \mathrm{~b}$ & $125,32 \% \mathrm{~b}$ \\
\hline
\end{tabular}

Médias seguidas por diferentes letras minúsculas diferem estatisticamente entre si.

Dureza Rockwell 
Os valores obtidos para a dureza Rockwell foram superiores nas madeiras acetiladas em relação àquele encontrado para a o material não tratado; contudo, não houve diferença significativa entre os tratamentos de 3 e 6 horas (Tabela 4).

Tabela 4. Comparação entre os valores médios de dureza Rockwell das madeiras de E. urophylla x E. grandis acetiladas e controle.

\begin{tabular}{cc}
\hline Tratamento & Dureza Rockwell \\
\hline Testemunha & 40,71 HRL a \\
3h & 71,57 HRL b \\
6h & 73,32 HRL b \\
\hline
\end{tabular}

Médias seguidas por diferentes letras minúsculas diferem estatisticamente entre si.

\section{Resistência ao ataque de cupins}

As madeiras submetidas ao tratamento de acetilação apresentaram maiores médias de notas comparadas às amostras controle, indicando que a acetilação proporciona maior resistência ao ataque de cupins do gênero Nasutitermes sp. (Tabela 5).

Tabela 5. Valores médios das notas atribuídas às madeiras de E. urophylla x E. grandis acetiladas e controle para a resistência ao ataque de Nasutitermes sp.

\begin{tabular}{cc}
\hline Tratamento & Nível de deterioração \\
\hline Testemunha & $5,3 \mathrm{a}$ \\
$3 \mathrm{~h}$ & $7,8 \mathrm{~b}$ \\
$6 \mathrm{~h}$ & $8,3 \mathrm{~b}$ \\
\hline
\end{tabular}

Médias seguidas por diferentes letras minúsculas diferem estatisticamente entre si.

\section{Discussão \\ Ganho de massa e massa específica aparente}

Os maiores valores para o ganho de massa foram observados no tratamento de acetilação por 6 horas, que denotou um aumento de 11,96\%, mostrando-se significativamente distinto dos demais tratamentos (Tabela 2). Os resultados obtidos corroboram com outros estudos realizados no âmbito da acetilação, que por sua vez apresentaram valores entre 8,7 e $20,9 \%$ para tratamentos com duração de 2 a 24 horas (Chauhan et al., 2001; Azeh et al., 2013; Castro et al., 2013).

O ganho de massa durante o processo de acetilação pode ser influenciado por alguns fatores, tais como a permeabilidade, composição química e dimensões da madeira, tempo e temperatura de tratamento e uso de catalisador (Rowell, 2014). Vale ainda pontuar que um maior tempo de reação proporciona uma maior substituição dos grupos hidroxílicos presentes na madeira, aumentando, consequentemente, o percentual de ganho de massa do material (Gröndahl et al., 2003).

Quanto à massa específica aparente, é possível observar que não houve diferença significativa entre os tratamentos (Tabela 2). Os resultados se mostram compatíveis com outros estudos que constataram o mesmo comportamento em partículas de madeira de folhosas como Eucalyptus grandis, Eucalyptus urophylla, Eucalyptus cloeziana e coníferas, como Pinus elliottii e Picea glehni (Akitsu et al., 1993; Cabral, 2005; Oliveira et al., 2010). Diante disso, pode-se afirmar que a acetilação não teve influência sobre a massa específica, fato compreendido pela constante proporcional entre o ganho de massa e o inchamento relativo ao tratamento.

\section{Absorção em água e inchamento volumétrico}

É perceptível que, quanto maior o ganho de massa do material após o tratamento de acetilação (Tabela 2), menor será a sua absorção em água (Tabela 3). Os resultados encontrados avigoram àqueles descritos em diversos estudos, que demonstraram que quanto maior o ganho de massa devido à acetilação, menor será a sua absorção em água e, consequentemente, menos pronunciado será o inchamento volumétrico do material (Gomes, 2005; Obataya e Gril, 2005; Temiz et al., 2006; Castro e Iwakiri, 2014).

De acordo com Castro e Iwakiri (2014), uma vez que a madeira passa pelo processo de acetilação, os grupos hidrófilos presentes na sua constituição passam a ser bloqueados, e com isso há uma menor afinidade entre a madeira acetilada e a água, resultando numa maior estabilidade dimensional.

\section{Dureza Rockwell}

As madeiras acetiladas apresentaram valores de dureza Rockwell superiores às amostras controle (Tabela 4), o que pode ser justificado pelo aumento na massa das madeiras tratadas.

De acordo com Papadopoulos e Tountziarakis (2011) em trabalho com a madeira acetilada de Pinus nigra, esta apresentou um ligeiro aumento da dureza Janka para os ganhos de massa de 5,3 e 14,7\% em comparação com as amostras não acetiladas. Contudo, para o ganho de massa de $22,8 \%$ houve redução na dureza em relação às amostras controle, porém estes resultados não foram significativos ao nível de confiança de $95 \%$. Conforme Cabral et al. (2006), em estudos realizados com chapas fabricadas com flocos de madeiras de Eucalyptus urophylla e Eucalyptus cloeziana, a acetilação proporcionou um aumento na dureza da superfície das chapas, que saíram de valores médios de $536 \mathrm{kgf}$ nas amostras não tratadas para $736 \mathrm{kgf}$ nas amostras acetiladas. Bongers e Beckers (2003), também demonstraram que as madeiras de Populus sp. e Pinus sylvestris acetiladas apresentaram aumento na dureza Janka, apresentando resultados semelhantes.

Segundo Homan et al. (2000), a dureza da madeira aumenta mediante o processo de acetilação, uma vez que a introdução de grupos acetatos 
proporciona um aumento na massa específica da parede celular.

\section{Resistência ao ataque de cupins xilófagos}

O tratamento de acetilação promoveu um aumento das notas atribuídas às amostras submetidas à deterioração, indicando uma melhora significativa da resistência biológica do material quando comparado à madeira não acetilada (Tabela 5).

Os resultados observados neste estudo são similares a outros estudos desenvolvidos acerca da resistência contra térmitas por parte das madeiras acetiladas, que pontuaram aumento gradativo desta propriedade na medida em que se intensifica o tratamento (Papadopoulos et al., 2008; Bongers et al., 2013; Alexander et al., 2014). Tais resultados se relacionam ao fato de que o aumento da dureza na madeira proporcionado pela acetilação é um fator condicionante ao ataque de térmitas, que por sua vez tendem a preferir madeiras com dureza reduzida e de menor densidade (Bongers et al., 2015).

\section{Conclusão}

A acetilação da madeira proporcionou aumento da estabilidade dimensional, resistência biológica e mecânica, quando comparada às amostras não tratadas. Por outro lado, não foi verificada alteração na massa específica aparente em condições anidras das madeiras acetiladas e controle.

O tempo de tratamento de acetilação de $3 \mathrm{~h}$ é o mais indicado, pois o intervalo de tempo superior testado não conferiu alteração das propriedades tecnológicas.

\section{Referências}

Akitsu H, Norimoto M, Morooka T, Rowell RM (1993) Effect of humidity on vibrational properties of chemically modified wood. Wood and Fiber Science, 25(3): 250-260.

Alexander J, Hague J, Roberts M, Imamura Y, Bongers F (2014) The resistance of Accoya ${ }^{\circledR}$ and Tricoya ${ }^{\circledR}$ to attack by wood-destroying fungi and termites. In: The International Research Group on Wood Protection. St. George, USA.

American Society for Testing and Materials - ASTM (1994) ASTM D-3345-08: Standard method for laboratory evaluation of wood and other cellulosic materials for resistance to termite. West Conshohocken: ASTM, 3p.

Associação Brasileira de Normas Técnicas - ABNT (1997) NBR 7190: Projeto de estruturas de madeira. Rio de Janeiro: ABNT, 107p.

Azeh Y, Olatunji GA, Mohammed C, Mamza PA (2013) Acetylation of wood flour from four wood species grown in Nigeria using vinegar and acetic anhydride. International Journal of Carbohydrate Chemistry, 2013(141034): 1-6p. doi:
10.1155/2013/141034.

Bongers HPM, Beckers EPJ (2003) Mechanical properties of acetylated solid wood treated on pilot plant scale. In: European Conference On Wood Modification. Ghent, Bélgica.

Bongers F, Hague J, Alexander J, Roberts M, Imamura Y, Suttie E (2013) The resistance of high performance acetylated wood to attack by wooddestroying fungi and termites. In: The International Research Group on Wood Protection. Estocolmo, Suécia.

Bongers F, Kutnik M, Paulmier I, Alexander J, Militz H (2015) Termite and insect resistance of acetylated wood. In: The International Research Group on Wood Protection. Viña del Mar, Chile.

Cabral CPT (2005) Propriedades de chapas tipo aglomerado e OSB, fabricadas com partículas e flocos de madeira de Eucalyptus grandis, Eucalyptus urophylla, Eucalyptus cloeziana e Pinus elliottii. 2005. Tese, Universidade Federal de Viçosa. 104p.

Cabral CPT, Vital BR, Della Lucia RM, Pimenta AS, Soares CPB, Carvalho AMML (2006) Propriedades de chapas tipo OSB, fabricadas com partículas acetiladas de madeiras de Eucalyptus grandis, Eucalyptus urophylla, Eucalyptus cloeziana e Pinus elliottii. Árvore, 30(4): 659-668. doi: 10.1590/S0100-67622006000400020.

Castro V, Klock U, Iwakiri S, Muniz GIB (2013) Avaliação colorimétrica de partículas de Pinus taeda submetidas a diferentes métodos de acetilação. Scientia Forestalis, 41(98): 265-270.

Castro V, Iwakiri S (2014) Influência de diferentes níveis de acetilação nas propriedades físicomecânicas de aglomerados e painéis madeiracimento. Cerne, 20(4): 535-540. doi: 10.1590/01047760201420041673.

Chauhan SS, Aggarwal P, Karmarkar A, Pandey KK (2001) Moisture adsorption behaviour of esterified rubber wood (Hevea brasiliensis). European Journal of Wood and Wood Products, 59(4): 250-253. doi: $10.1007 / \mathrm{s} 001070000152$.

Curling S, Winandy JE, Clausen CA (2000) An experimental method to simulate incipient decay of wood by basidiomycete fungi. In: The International Research Group on Wood Protection, Kona, USA. $13 \mathrm{p}$.

Esteves B, Pereira H (2009) Novos Métodos de Protecção da Madeira. In: Congresso Florestal Nacional, Ponta Delgada, Portugal. 
Esteves B, Machado JS, Nunes L (2011) Utilização de madeira modificada na construção. In: Congresso Ibero-Latino Americano de Madeira na Construção, Coimbra, Portugal.

Gomes DFF (2005) Avaliação da estabilidade dimensional da madeira de Eucalyptus grandis Hill ex. Maiden após acetilação. Dissertação, Universidade Federal de Lavras. 85p.

Gröndahl M, Teleman A, Gatenholm P (2003) Effect of acetylation on the material properties of glucuronoxylan from aspen wood. Carbohydrate Polymers, 52(4): 359-366. doi: 10.1016/S01448617(03)00014-6.

Homan WJ, Tjeerdsma B, Beckers E, Jorissen A (2000) Structural and other properties of modified wood. In: World Conference on Timber Engineering, British Columbia, Canadá.

Homan WJ, Bongers F (2004) Influence of upscaling processes on degree and gradient of acetylation in Spruce and Beech. In: COST Action E, Estoril, Portugal.

Obataya E, Gril J (2005) Swelling of acetylated wood I. Swelling in organic liquids. Journal of Wood Science, 51(2): 124-129. doi: 10.1007/s10086-0040634-2.

Oliveira FV, Vital BR, Castro Silva JD, Carneiro AD, Pimenta AS (2010) Efeito da acetilação das partículas de madeira de Eucalyptus grandis e da inclusão de partículas de polietileno e de embalagens cartonadas nas propriedades de chapas de aglomerado. Revista Árvore, 34(5): 937-946. doi: 10.1590/S0100-67622010000500019.

Papadopoulos AN, Avtzis DN, Avtzis ND (2008) The biological effectiveness of wood modified with linear chain carboxylic acid anhydrides against the subterranean termites Reticulitermes flavipes. Holz als Roh-und Werkstoff, 66(4): 249-252. doi: 10.1007/s00107-008-0252-6.

Papadopoulos AN, Tountziarakis P (2011) The effect of acetylation on the Janka hardness of pine wood. European Journal of Wood and Wood Products, 69(3): 499-500. doi: 10.1007/s00107-0100484-0.

Rowell RM (2013) Handbook of wood chemistry and wood composites. Boca Raton: CRC Press, $703 p$.

Rowell RM (2014) Acetylation of wood - a review. International Journal of Lignocellulosic Products, 1(1): 1-27. doi: 10.22069/IJLP.2014.1920.
Stangerlin, DM, Costa, AF, Pastore, TCM, Garlet, A. (2013). Dureza Rockwell da madeira de três espécies amazônicas submetidas a ensaios de apodrecimento acelerado. Ciência Rural, 43(4): 623630. doi: 10.1590/S0103-84782013005000022.

Temiz A, Terziev N, Jacobsen B, Eikenes M (2006) Weathering, water absorption, and durability of silicon, acetylated, and heat treated wood. Journal of Applied Polymer Science, 102(5): 4506-4513. doi: 10.1002/app. 24878.

Valverde SR, Soares NS, Silva MD, Jacovine LAG, Neiva DAS (2004) O comportamento do mercado da madeira de eucalipto no Brasil. Biomassa \& Energia, 1(4): 393-403. 